\title{
LAS NUEVAS INTUICIONES CONCEPTUALES DE JOHN MCDOWELL ${ }^{1}$
}

\author{
John McDowell's new conceptual intuitions
}

Francisco Pereira Gandarillas*

\begin{abstract}
RESUMEN
En este artículo contrastaré la propuesta conceptualista estándar que McDowell defendió en Mente y Mundo con su actual teoría conceptualista que distingue entre dos clases de contenido: el contenido intuitivo no proposicional de la experiencia perceptual y el contenido proposicional discursivo de nuestras creencias empíricas. Argumentaré que, aunque McDowell caracteriza ambas propuestas como "conceptualistas", de hecho su propuesta actual es conceptual en un sentido menos demandante, ya que carece de los compromisos ontológicos que la versión clásica de Mente y Mundo tenía respecto de los componentes de los contenidos de la percepción. La variedad de conceptualismo sobre el contenido de la percepción que McDowell hoy defiende incluso admitiría la presencia de algunos elementos constitutivos de naturaleza no conceptual.
\end{abstract}

Palabras clave: McDowell, conceptualismo, percepción, contenido intuitivo, contenido proposicional.

${ }^{1}$ El autor agradece el financiamiento otorgado por el Proyecto Regular Fondecyt No 1141002, Gobierno de Chile.

* Departamento de Filosofía, Universidad Alberto Hurtado. Santiago, Chile. Correo electrónico: fpereira@uahurtado.cl

Artículo recibido el 04 de marzo de 2015. Aceptado el 26 de octubre de 2015. 


\begin{abstract}
In this paper I will contrast the standard conceptualist account that McDowell put forward in Mind and World with his current conceptualist theory that distinguishes between two kinds of content: the non-propositional intuitive content of perceptual experiences and the discursive propositional content of our empirical beliefs. I will argue that, even though McDowell characterizes both theories as "conceptualist", in fact his current proposal is conceptual in a less demanding sense than the previous one, because it lacks the ontological commitments that the classic version of Mind and World had regarding the constituents of the contents of perception. The variety of conceptualism regarding the content of perception that McDowell nowadays supports would even admit the presence of some non-conceptual constitutive features.
\end{abstract}

Keywords: McDowell, conceptualism, perception, intuitive content, propositional content.

\title{
INTRODUCCIÓN
}

Una de las propuestas más influyentes de la epistemología y de la filosofía de la mente contemporáneas es la que John McDowell desarrolla en su ya célebre obra Mente y Mundo. Allí McDowell considera la plausibilidad de un "empirismo mínimo" (2003: 15), es decir, de una propuesta epistemológica que concibe la experiencia perceptual como el origen y fundamento de la justificación de todas nuestras creencias acerca del mundo empírico. La plausibilidad del empirismo mínimo consta de dos características fundamentales. Primero, la experiencia se entiende aquí como una instancia que efectivamente juega un rol en los procesos de justificación epistémica de nuestras creencias empíricas. Las experiencias justifican nuestras creencias empíricas, son razones para creer que tal y tal es el caso. Segundo, la posibilidad misma de los contenidos de nuestros pensamientos acerca del mundo empírico radica en el hecho de que estos deben ser responsables ante la experiencia (McDowell, 2003: 18), la cual se constituye como un tribunal. El contenido de una creencia o de una percepción, el hecho de que seamos capaces de representar el mundo correcta o incorrectamente, se funda en la normatividad propia de los vínculos efectivos entre la mente y el mundo.

El empirismo mínimo de McDowell se opone directamente a dos concepciones muy arraigadas en la teoría del conocimiento. Por una parte, al aceptar que nuestras experiencias perceptivas efectivamente le otorgan justificación a nuestras creencias empíricas, McDowell se distancia de una propuesta coherentista clásica de la justificación epistémica, tal como la que defiende Davidson (2001), en la que solo una creencia puede contar como razón para otra creencia. Para el coherentismo davidsoniano la relación entre pensamiento y mundo se da en un plano estrictamente 
causal, las experiencias causan las creencias empíricas, pero no las justifican. Por el contrario, desde la perspectiva de McDowell, cualquier forma de pensamiento empírico requiere de una fricción o constreñimiento racional externo por parte de la experiencia. Así lo ha manifestado, por ejemplo, al decir que "Davidson dibuja nuestro pensamiento como algo que no cuenta con ninguna constricción racional, sino sólo con influencias causales" (2003: 52) y al recordarnos que "el pensamiento debe ser responsable ante las impresiones si ha de tener algún contenido en absoluto" (2009b: 248).

Por otra parte, McDowell también se distancia de aquellas doctrinas epistemológicas de inspiración fundacionalista que aceptando diversas formas de dualismo -el pensamiento articulado conceptualmente y lo dado por la experienciasostienen que el espacio de las razones y de la justificación se extiende más allá de la esfera de lo conceptual. A juicio de McDowell, esta pretensión de extender la justificación a un dominio donde hipotéticamente la espontaneidad conceptual es inoperante -al espacio lógico de las causas naturales-constituyen un mito, el Mito de lo Dado:

El problema con el Mito de lo Dado era que nos proporcionaba, como mucho, exculpaciones donde lo que queríamos era justificaciones... Si tales entradas se conciben como algo que queda fuera del campo de la espontaneidad, fuera del dominio de la libertad responsable, entonces lo máximo que pueden suministrarnos es un motivo para que no se nos culpe por creer cualquier cosa que tales entradas nos hagan creer, y no un motivo para que nos esté justificado el creer tal cosa (McDowell, 2003: 50-51).

Para proponer una solución epistemológica que no nos haga oscilar entre posiciones de orden coherentista y el Mito de la Dado, McDowell va a inspirarse en el proyecto kantiano, particularmente en la idea de un conocimiento empírico que resulta de una colaboración efectiva y constitutiva entre receptividad y espontaneidad (2003: 87-88). El conocido y difícil pasaje de la Crítica de la Razón Pura que McDowell menciona en Mente y Mundo ilustra adecuadamente su anhelos trascendentales:

Si llamamos sensibilidada la receptividad de nuestra mente para recibir representaciones en la medida en que de alguna manera es afectada, entonces, en cambio, la facultad de producir ella misma representaciones, o la espontaneidad del conocimiento, es el entendimiento. Es propio de nuestra naturaleza el que la intuición nunca puede ser sino sensible, es decir, sólo contiene la manera como somos afectados por los objetos. Por el contrario, la facultad de pensar el objeto de la intuición sensible es el entendimiento. Ninguna de estas propiedades ha de preferirse a la otra. Sin sensibilidad no nos sería dado objeto alguno; y sin entendimiento, ninguno sería pensado. Pensamientos sin contenidos son vacíos, intuiciones sin conceptos son ciegas (Kant, 2007: A51/B75). 
La lectura que McDowell hace de Kant asume que para este último las intuiciones no podrían jugar un rol cognitivo importante en la experiencia si no fuera por el necesario involucramiento de las capacidades conceptuales que paradigmáticamente son ejercitadas en nuestros juicios empíricos. En este sentido, la experiencia misma y sus contenidos representacionales deben pensarse como configuradas conceptualmente -como perteneciendo al espacio lógico de las razonesteniendo solo así la capacidad de vincularse racionalmente con los contenidos de las creencias fundadas en dichas experiencias ${ }^{2}$.

Podemos apreciar con claridad que una de las motivaciones fundamentales del proyecto de McDowell es epistemológica. Los contenidos de nuestras creencias empíricas obtienen justificación racional en los contenidos de la experiencia y son responsables ante ellos. En ambos casos -experiencia perceptiva y creencia empíricala forma en que representamos el mundo está inserta en un dominio donde nuestras capacidades conceptuales operan. En lo que resta de este artículo presentaré la forma en que originalmente McDowell articula su propuesta conceptualista acerca de la experiencia en Mente y Mundo, para evaluar luego el importante giro que ha dado recientemente (2007a; 2007b; 2009a) y las consecuencias que a mi juicio se siguen de esta transformación. El énfasis estará puesto en dos elementos fundantes de su propuesta epistemológica: el carácter proposicional de los contenidos de la experiencia sensible y su naturaleza conceptual. Intentaré mostrar que McDowell, al transitar de una lectura proposicional en Mente y Mundo (2003) a una lectura no-proposicional de la experiencia en sus últimos trabajos, modifica también de manera importante la clase y el alcance del conceptualismo que defiende, permitiendo incluso hoy que los contenidos de la percepción incluyan como componentes algunos elementos de naturaleza no-conceptual.

\section{MENTE Y MUNDO: EL MCDOWELL CLÁSICO}

En su obra Mente y Mundo (2003), McDowell se compromete con una formulación estándar de la tesis del conceptualismo perceptual. Según esta tesis filosófica, en todos los casos el contenido de una experiencia perceptiva está necesariamente constituido por conceptos que especifican a cabalidad cada uno de los

\footnotetext{
${ }^{2}$ En Mente y Mundo, McDowell afirma que las capacidades conceptuales ya están implementadas de forma pasiva en la receptividad, teniendo siempre claro que el operar pasivo de estas capacidades conceptuales no se puede comprender con independencia de su implementación activa en los juicios acerca del mundo empírico. De hecho, McDowell parece llevar esta hipótesis kantiana al límite, afirmando que "la idea misma de contenido representacional, y no sólo la idea de juicios que estén justificados apropiadamente, requiere de la interacción entre conceptos e intuiciones" (2003: 41).
} 
elementos disponibles a nivel consciente durante la experiencia de modo simultáneo. McDowell (2003) se inserta así en la tradición neo-fregeana y defiende una teoría fuerte en que los contenidos perceptuales poseen exhaustivamente una estructura proposicional cuyos componentes son conceptos o "modos de presentación" cuyas condiciones de posesión están especificadas en términos de la posesión e implementación de habilidades que de hecho satisfacen cabalmente el llamado Requerimiento de Generalidad propuesto originalmente por Evans (1982)4.

El conceptualismo de McDowell tiene una clara motivación epistemológica. Al igual que otros conceptualistas (Brewer, 1999; 2005), su propuesta clásica comienza aceptando que las experiencias perceptivas justifican nuestras creencias empíricas y concluye que si esto es así entonces toda experiencia perceptiva debe necesariamente ser proposicional y, por lo mismo, exhaustivamente conceptual. La transición desde una premisa abiertamente anti-coherentista -la experiencias otorgan razones o justifican creencias- a una conclusión acerca de la naturaleza del contenido experiencial -los contenidos de la experiencia son conceptuales- solo tiene sentido si ampliamos la cobertura del espacio lógico de las razones e incluimos a las experiencias mismas y sus contenidos en este dominio. Así, McDowell sugiere modificar el lema davidsoniano, según el cual solo una creencia puede ser una razón para otra creencia, por la tesis de inspiración kantiana que afirma que "nada puede contar como una razón para sostener una creencia excepto alguna otra cosa que también se encuentre en el espacio de los conceptos" (McDowell, 2003: 223).

\footnotetext{
3 Es precisamente esta exhaustividad -el hecho de que todos los elementos representados simultáneamente por una experiencia perceptiva posean una naturaleza conceptual- la que caracteriza distintivamente la perspectiva metafísica inherente al proyecto proposicional conceptualista de Mente y Mundo. La herencia neo-fregeana por sí misma no implica necesariamente el conceptualismo tal como lo entiende aquí McDowell (2003), ya que muchos teóricos que defienden la posibilidad de contenidos no-conceptuales de hecho son parte de la misma tradición (Peacocke, 2001; Evans, 1986). ${ }^{4}$ Evans afirma que "no podemos evitar pensar un pensamiento acerca de un objeto individual $\mathrm{x}$, para efectos de que $x$ es $F$, como el ejercicio de dos capacidades distintas; una siendo la capacidad de pensar sobre $x$, la cual puede ser igualmente ejercitada en pensamientos acerca de $x$ para efectos de que $x$ es $G$ o $H$; y la otra siendo una concepción acerca de qué es ser $F$, la cual podría ser igualmente ejercitada en pensamientos acerca de otros objetos individuales, para efectos de que estos son $F$ " (1982:75). McDowell (2003) y Brewer (1999) que han seguido de cerca a Evans parecen estar de acuerdo en que el tener o estar en posesión de un concepto involucra al menos la habilidad de identificar y re-identificar cosas y propiedades que caen bajo ese concepto, la habilidad para realizar inferencias utilizando el concepto, la habilidad para aplicar el mismo concepto a diferentes cosas y la habilidad para discriminar entre aquellas cosas que caen bajo aquel concepto de las que no lo hacen, entre otras habilidades o capacidades. Un sujeto que posee un concepto $F$ debe ser capaz de implementar las habilidades correspondientes en una amplia gama de pensamientos acerca de $F$. El sujeto debe ser capaz de pensar que $x$ es $F$, que y es $F$, e incluso que $z$ no es $F$. Por lo tanto, no es posible atribuirle a un sujeto la posesión de un concepto a no ser que este sujeto muestre algún grado de generalidad al nivel de la implementación.
} 
Contra el Mito de lo Dado, McDowell sostiene que nuestros juicios de experiencia no conceptualizan algo no conceptual previamente otorgado por la sensibilidad de un modo pasivo. Por el contrario, las capacidades conceptuales ya están en acción configurando lo que la sensibilidad nos proporciona. Contra el coherentismo davidsoniano, la experiencia efectivamente otorga justificación para nuestros juicos empíricos y constrińe normativamente nuestro pensamiento como un tribunal ante el cual somos responsables. El conceptualismo sin límites de McDowell, al ser condición de posibilidad de la experiencia representacional misma, garantiza que la constricción llegue "desde fuera del pensamiento, pero no desde fuera de lo que es pensable” (McDowell, 2003: 71).

Dado que las experiencias perceptivas otorgan razones/justifican nuestros juicios empíricos, el contenido de dichas experiencias debe ser de la misma clase o tipo que el contenido de nuestros juicios, es decir, un contenido proposicional constituido por conceptos (neo) fregeanos. Es de suma importancia enfatizar que lo que está en juego aquí es una tesis fuerte de carácter ontológico acerca de la naturaleza de los contenidos de la percepción y no meramente una tesis débil acerca de los estados mentales en los que se encuentra el agente y la relación que estos puedan tener con los contenidos proposicionales. Particularmente, estamos afirmando que para McDowell los contenidos de la percepción y los contenidos de las creencias que formamos sobre la base de las percepciones son necesariamente del mismo tipo: proposicionales y compuestos de conceptos. Con estos dos aspectos fundamentales ya considerados es importante que reparemos en dos elementos adicionales del proyecto filosófico de Mente y Mundo: la reflexividad interna que a juicio de $\mathrm{McD}$ owell requieren todos los procesos de justificación y el aparente carácter inferencial o cuasi-inferencial de la relación de justificación entre experiencia y creencia empírica.

Primero, una experiencia solo puede constreñir racionalmente y ser razón para un juicio o creencia empírica si efectivamente es considerada como tal desde la perspectiva del sujeto. Esto evidentemente es un compromiso epistémico de carácter internista, según el cual la justificación de una creencia superviene en hechos accesibles solo por reflexión desde la perspectiva de la primera persona. Tal como sugiere Toribio, aquí la reflexión "incluye conciencia introspectiva de los propios estados mentales del sujeto, conocimiento a priori y memoria de todo el conocimiento hasta entonces adquirido" (2008: 3$)^{5}$. Segundo, el modelo con el

\footnotetext{
${ }^{5}$ Este gesto reflexivo internista acerca de la justificación epistémica configura la epistemología mcdowelliana hasta hoy. Lo mismo sucede en el caso de otros conceptualistas que lo siguen de cerca, como Brewer que asegura que las razones que tiene un sujeto para creer que $p$ "deben ser accesibles cognitivamente" (1999: 120) al sujeto de experiencia y ser "necesariamente reconocidas como tales" (1999: 165).
} 
que parece estar comprometido McDowell en Mente y Mundo es un modelo de la justificación racional de índole inferencial en el que los estados experienciales provistos de contenido proposicional figuran como premisas de argumentos cuyas conclusiones son las creencias o juicios empíricos acerca del mundo. Si bien el mero hecho de que tanto el contenido de las creencia empíricas, como el de las experiencias perceptivas que las justifican sean proposicionales no garantiza de inmediato que este tipo de transición sea inferencial, lo cierto es que el mismo McDowell tiempo después reconoció, independientemente de cuál eran sus intenciones genuinas, que un modelo como el de Mente y Mundo, "puede interpretarse como recomendando una concepción inferencial o al menos cuasi-inferencial de la manera en que la experiencia garantiza nuestras creencias perceptivas" (McDowell, 2009a: 270) .

Las consideraciones anteriores nos pone en condición de apreciar adecuadamente los compromisos fundamentales de la propuesta epistemológica clásica que McDowell ofrece en Mente y Mundo. Su teoría clásica se compromete con cuatro elementos sin los cuales supuestamente nuestras experiencias perceptuales no podrían justificar nuestras creencias empíricas y menos aun constreñir racionalmente nuestros juicios acerca del mundo. Primero, el contenido de las experiencias es un contenido proposicional. Segundo, dado que las proposiciones están compuestas de conceptos o sentidos neo-fregeanos es necesario aceptar que los contenidos de las experiencias perceptivas -en caso de ser proposicionales como McDowell afirma en Mente y Mundo- son de una naturaleza exhaustivamente conceptual. Tercero, la idea misma de justificación epistémica comprendida en términos racionales exige accesibilidad cognitiva por parte del sujeto. Es decir, las experiencias son razones para nuestras creencias solo en la medida que el sujeto las puede apreciar como tal. Cuarto, dado que los contenidos de las experiencias y de las creencias que formamos en base a dichas experiencias son necesariamente proposicionales, es perfectamente sensato interpretar el modelo de justificación empírica propuesto por McDowell como un modelo inferencial o cuasi-inferencial de la justificación ${ }^{7}$.

\footnotetext{
${ }^{6}$ Wright, por ejemplo, leyendo a McDowell afirmó explícitamente que este modelo constituye una "una concepción cuasi-inferencial de la justificación empírica" (1998: 400) y Brewer, uno de los fieles seguidores del McDowell clásico, afirma que "estados experienciales sensoriales proveen razones para las creencias empíricas solo en virtud de sus relaciones apropiadas con proposiciones relacionadas inferencialmente de manera pertinente a los contenidos de las creencias en cuestión" (2005: 219).

${ }^{7}$ Una forma tradicional de comprender en qué consiste una forma de justificación inferencia o cuasi-inferencial es afirmar que se trata de una forma de justificación mediada. Por ejemplo, si la justificación que tiene un sujeto para creer que P viene en parte dada por la justificación que este mismo sujeto tiene para creer otras proposiciones que proporcionan soporte epistémico, entonces estamos en presencia de una clase de justificación que no es inmediata o no-inferencial (Pryor, 2005).
} 
Este modelo proposicional, conceptual, internista y aparentemente inferencial posee algunas debilidades evidentes y ha sido ampliamente discutido en la literatura filosófica de los últimos 15 años $^{8}$. Sin embargo, en las secciones que vienen repararemos solo en aquellos elementos que parecen haber atribulado al mismo McDowell y que recientemente han provocado un cambio en su posición filosófica. Un análisis minucioso de estos elementos nos permitirá comprender con mayor claridad la nueva posición de McDowell y evaluar dónde se ubica ahora su propuesta conceptualista de inspiración kantiana que él mismo popularizó en la década de los noventa.

\section{EL NUEVO MCDOWELL}

John McDowell recientemente ha cambiado su posición acerca de la relación existente entre nuestras experiencias perceptuales y nuestras creencias empíricas acerca del mundo. En sus Gareth Evans Memorial Lectures dictadas en la Universidad de Oxford el año 2007 y publicadas en diferentes versiones (2007c; 2009a) se distancia de algunos de los compromisos claves de Mente y Mundo'. En uno de los pasajes claves de esta obra, hoy mejor conocida bajo el título Avoiding the Myth of the Given, McDowell se pronuncia explícitamente acerca de algunos errores cometidos en el pasado:

Yo solía suponer que para concebir las experiencias como actualizaciones de capacidades conceptuales, necesitaríamos acreditar las experiencias con contenido proposicional, la clase de contenido que tienen los juicios. Y solía asumir que

\footnotetext{
${ }^{8}$ Una de estas debilidades dice relación con la aparente imposibilidad que hay de atribuirle experiencias con contenido a animales e infantes que no tienen las habilidades conceptuales requeridas por el modelo conceptualista (Bermúdez, 1994). Otra debilidad se fundaría en el hecho de que nuestras experiencias perceptuales representarían el mundo con un grado de fineza (Peacocke, 2001) o de riqueza (Tye, 2006) que no puede capturarse adecuadamente por una estructura conceptual del contenido experiencial. También se han planteado algunas objeciones directas al modelo provenientes de la psicología cognitiva. En particular, existiría evidencia empírica según la cual habrían formas de representar el mundo que en ningún caso requieren de la implementación de conceptos (Lamme, 2003; Raftopoulus 2009). Finalmente, desde una perspectiva epistémica la tesis internista a la cual adhiere McDowell es, a juicio de algunos comentaristas, excesivamente intelectual y restrictiva a la hora de hacer atribuciones epistémicas. Estos son solo algunos de los problemas que han configurado el debate entre conceptualistas y no-conceptualistas acerca del contenido de la experiencia perceptiva o entre internistas y externistas en el ámbito de la justificación epistémica en el último tiempo.

${ }^{9}$ Sin duda hay intercambios filosóficos que han motivado o determinado algunos de estos cambios. Entre ellos cabe destacar el rol que han jugado las críticas a su doctrina por parte de Travis (2004; 2007) y Dreyfus (2005; 2007a; 2007b).
} 
el contenido de una experiencia necesitaría incluir todo lo que la experiencia le permite a un sujeto conocer de manera no inferencial. Pero ambos supuestos me parecen ahora equivocados (McDowell, 2009a: 258).

En este pasaje McDowell reconoce dos diferencias fundamentales con su antigua doctrina, además de otorgar una aclaración acerca del tipo de relación de justificación -una relación no-inferencial- existente entre los contenidos de la experiencias y los contenidos de los juicios o creencias fundados en dichas experiencias. En primer lugar, McDowell reniega de una de las características fundamentales de su tesis clásica al negar que el contenido de la experiencia sea un contenido proposicional. En segundo lugar, McDowell aclara que el contenido de la experiencia no necesita contener necesariamente todo lo que el sujeto es capaz de conocer no-inferencialmente sobre la base de dicha experiencia. Estos dos compromisos podrían confundirnos y llevarnos a pensar que él no solo ha dejado a un lado el requisito proposicional (elemento central de las tesis conceptualistas estándar), sino que además ha desechado cualquier forma de conceptualismo. Sin embargo, él mismo sugiere que esto sería un serio error:

Deberíamos desechar la idea misma de que las experiencias perceptuales que tienen los animales racionales tienen contenido conceptual? Esto sería demasiado drástico... las capacidades que pertenecen a la facultad cognitiva superior deben estar operativas en la experiencia... (McDowell, 2009a: 260).

McDowell sostiene las capacidades conceptuales tienen un importante rol que jugar en la experiencia. De lo contrario, él correría el riesgo de caracterizar la experiencia como algo que no requiere de la implementación de conceptos transformándose, bajo sus propios estándares, en un exponente del Mito de lo Dado. Agregando a esta idea las modificaciones mencionadas en el pasaje anterior podemos ver que McDowell se compromete con cuatro tesis (T1 - T4) fundamentales en su nueva propuesta:

(T1) El contenido de las experiencias perceptuales no es proposicional.

(T2) El contenido de las experiencias perceptuales no está compuesto por todo aquello que el sujeto de dichas experiencias puede conocer sobre su base.

(T3) El contenido de las experiencias perceptuales no es idéntico al contenido de las creencias o juicios de conocimiento que formamos sobre la base de dichas experiencias.

(T4) En toda experiencia perceptual están en juego capacidades conceptuales.

La nueva teoría de McDowell sostiene que los contenidos de las experiencias perceptuales no están estructuradas proposicionalmente (T1). Además dichos 
contenidos no requieren estar compuesto o constituido por todo aquello que somos capaces de conocer sobre la base de dichas experiencias. En otros términos, no todos los elementos conceptuales constitutivos de las estructuras proposicionales características del conocimiento, la creencia o el juicio están presentes en la experiencia (T2). Finalmente, dado T1 y T2 es evidente que McDowell se compromete ahora con una teoría según la cual no debemos identificar el contenido de las experiencias con el contenido de los juicios que formamos sobre la base de dichas experiencias (T3) ${ }^{10}$. Sin embargo, el hecho de que los contenidos no sean idénticos no implican que estos no pertenezcan al ámbito de lo conceptual, es decir, al dominio en el que operan capacidades esencialmente ligadas a nuestras actitudes proposicionales (T4). Cada una de estas tesis requiere de un cuidadoso análisis o, por lo menos, de una justificación.

Comenzando con T1 cabe preguntar por qué McDowell sostiene ahora que no debemos atribuirle a la experiencia un contenido proposicional. Recordemos que la motivación fundamental del McDowell clásico a la hora de asignarle un contenido proposicional a la experiencia era de orden epistémico. Todo tipo de relación normativa, incluyendo para $\mathrm{McDowell}$ ciertamente la de justificación, debe comprenderse como una relación entre eventos o entidades insertas en el espacio lógico de las razones. Las experiencias perceptivas solo podían entrar en relaciones normativas de justificación -ser razones para- con nuestras creencias empíricas si sus contenidos eran de la misma clase. Algo no articulado proposicionalmente y al mismo tiempo, no compuesto de conceptos, no podía figurar de forma alguna como relata de una relación racional de justificación.

Uno podría estar satisfecho con esta estrategia proposicional que caracteriza la experiencia perceptual como una forma de interacción con un mundo tractariano de hechos, pero sin duda hay buenas razones para cuestionarla. Por ejemplo, uno podría sospechar de cualquier lectura que caracterice nuestras experiencias perceptuales como involucrando esencialmente relaciones con entidades abstractas como las proposiciones, particularmente si estas pertenecen a un dominio abstracto del sentido fregeano. No es que las proposiciones no existan, sino que entidades con ese nivel de abstracción que presumiblemente no están localizadas en el tiempo y en el espacio no parecería jugar rol alguno en la caracterización de la experiencia consciente (Papineau, 2014).

\footnotetext{
${ }^{10} \mathrm{Si}$ conocer es una actitud proposicional, entonces el contenido del conocimiento no puede ser identificado con el contenido de la experiencia. Por ejemplo, si John sabe que una lechuza vuela sobre el bosque de eucaliptus claramente John debe poseer e implementar los conceptos LECHUZA, EUCALIPTUS y VOLAR. Sin embargo, tal como afirma McDowell (2009a), dichos conceptos no tienen por qué componer el contenido de la experiencia que justifica de forma no inferencial dicho juicio.
} 
Sin embargo, sospechas de este tipo, las que normalmente están vinculadas a los proyectos naturalistas claramente no son las que un filósofo como McDowell tiene en mente. Su motivación particular está aparentemente relacionada con algo mucho más preciso y en directa vinculación con algunas de las críticas que Travis (2004; 2007) elaboró a la propuesta de Mente y Mundo. El problema al que me refiero apela al hecho de que las representaciones proposicionales que hacemos del mundo en la experiencia y su corrección/verdad o incorrección/falsedad parece depender o ser sensibles a factores contextuales y a los criterios que se utilicen en una ocasión en particular para evaluar los mismos estados de cosas. Normalmente el contenido proposicional de una experiencia perceptual se fija sobre la base de cómo las cosas lucen o parecen ser en un momento determinado. El cómo se ven, por ejemplo, las cosas desde la perspectiva del sujeto fija el contenido de la experiencia visual que ese sujeto tiene en esa ocasión. Sin embargo, parecen existir formas diferentes de representar proposicionalmente lo que percibimos en el mundo sobre la base de cómo lucen las cosas igualmente correctas.

Lo anterior desencadena dos problemas importantes. En primer lugar, no parece existir claridad respecto de qué es exactamente lo que determina en una ocasión en particular la o las proposiciones que de hecho se nos dan en la experiencia. En segundo lugar, si efectivamente se nos da un contenido proposicional específico como el contenido de una percepción en una ocasión específica, parece muy difícil que ese mismo contenido particular sea capaz de justificar contenidos alternativos, pero igualmente correctos, que se nos podrían haber dado en esa misma ocasión ${ }^{11}$. Además, en una ocasión exactamente la misma proposición respecto del mismo estado de cosas podría ser falsa, mientras que en otro contexto verdadera. Por ejemplo, en el contexto de una cena en la cual gran parte de los asistentes come alimentos vegetales parece correcto decirle a una mujer "veo que comes carne" mientras ella come riñones. Sin embargo, si el resto de los asistentes estuviera de hecho consumiendo sendos trozos de lomo vetado, sería cuestionable la corrección del mismo contenido proposicional.

La imposibilidad de establecer criterios adecuados para especificar qué contenido proposicional se nos da en la experiencia y la existencia de contenidos proposicionales alternativos y correctos sobre la base de una experiencia en la que figuran la misma clase de particulares concretos parece cuestionar el modelo según

\footnotetext{
${ }^{11}$ Travis argumenta que el cómo las cosas lucen en un momento determinado es algo íntimamente ligado al contexto y a los criterios de evaluación que se implementan. Esto es particularmente relevante, por ejemplo, cuando realizamos comparaciones sobre la base de cómo las cosas lucen, ya que si estas son verdaderas o no va a depender de cómo se hacen o bajo qué criterio se legitiman. Por ejemplo, "Pía lucirá como Pía en diez años cuando el asunto es visto de una manera, pero lamentablemente no cuando se ve de otra forma" (Travis, 2004: 71).
} 
el cual al percibir, aquello que constituye exhaustivamente el contenido de nuestra percepción es siempre que tal y tal es el caso. La nueva propuesta de McDowell no es ajena a esta clase de preocupaciones y parece buscar una caracterización más básica y menos intelectualizante respecto de cómo y qué percibimos. Evaluando las críticas de Travis (2004; 2007), McDowell sostiene ahora que el contenido de la experiencia no es un contenido proposicional, sino un contenido intuitivo (apelando a la noción kantiana de Anschaunng) que no es articulado y que debe ser claramente distinguido del contenido discursivo de los juicios o creencias que formamos sobre la base de nuestras experiencias sensibles que sí son siempre articulados y proposicionales. En su teoría clásica él supuso que las experiencias perceptivas que involucraban el reconocimiento de objetos comunes debían necesariamente tener como contenidos proposiciones articuladas que incluyeran como componentes esenciales los conceptos para aquellos objetos. Para el nuevo McDowell (2009a) el contenido no es proposicional (T1), entre otras razones porque el contenido de las experiencias perceptuales no está compuesto por todo aquello que el sujeto de dichas experiencias puede conocer sobre en base a ellas (T2):

Supongan que tengo un pájaro a plena vista, y eso me pone en posición para conocer de manera no inferencial que es un cardenal... lo que parece correcto es esto: $\mathrm{mi}$ experiencia hace que el pájaro se me presente visualmente, y mi capacidad de reconocimiento me permite conocer de forma no inferencial que aquello que veo es un cardenal. Incluso si seguimos asumiendo que mi experiencia tiene contenido, no hay necesidad de suponer que el concepto bajo el cual mi capacidad de reconocimiento me permite traer aquello que yo veo figure en aquel contenido (McDowell, 2009a: 259).

La experiencia perceptual de ver un cardenal puede efectivamente ser la misma si es que uno de hecho es capaz de reconocer un cardenal o no, es decir, puede ser la misma independientemente de si uno es capaz de implementar el concepto CARDENAL para la entidad particular que observamos. El sujeto que posee el concepto CARDENAL y que juzga que hay un cardenal sobre la rama de aquel árbol efectivamente se encuentra en un estado de actitud proposicional con el contenido hay un cardenal sobre la rama de aquel árbol. Sin embargo, el contenido proposicional de su juicio no debe confundirse con el contenido de la percepción. Según la nueva propuesta filosófica de McDowell, el contenido de la percepción no solo no es proposicional, sino que además no necesita incluir el concepto CARDENAL. Fundados en estas afirmaciones de McDowell tenemos los elementos suficientes para atribuirle T1 (el contenido de la experiencia no es proposicional), T2 (el contenido de las experiencias perceptivas no está compuesto por todo aquello que el sujeto de dichas experiencias puede conocer sobre su base) 
y ciertamente T3 (el contenido de la experiencias perceptuales no es idéntico al contenido de las creencias o juicios de conocimiento que formamos sobre la base de dichas experiencias).

¿Cuál es el estatuto de T4? Si bien McDowell afirma que el contenido perceptual no tiene por qué incluir todos los conceptos que conforman el contenido proposicional del conocimiento que un sujeto puede adquirir sobre la base de una experiencia, él nos recuerda que no todos los conceptos que figuran en el contenido proposicional del conocimiento pueden ser excluidos, en particular "las capacidades conceptuales asociadas con los conceptos de sensibles propios y comunes" (McDowell, 2009a: 260) ${ }^{12}$. Estas capacidades conceptuales "deben estar en juego en la experiencia si deseamos evitar el Mito", ya que son "capacidades que pertenecen a la racionalidad en un sentido demandante" (McDowell, 2009a:262). Esto sugiere prima facie que McDowell ha debilitado en algún sentido su conceptualismo, pero debemos ser cuidadosos.

¿Qué quiere decir exactamente que las capacidades conceptuales "estén en juego" en la experiencia? Por una parte, el contenido intuitivo no tiene las mismas características que el contenido discursivo de los juicios que nosotros realizamos sobre la base de dichas experiencias. Estos juicios poseen un contenido que es articulado y proposicional. Esto podría llevarnos a pensar que el contenido intuitivo de la experiencia, al no ser articulado y menos aún proposicional, podría ser un contenido no conceptual. Sin embargo, por otra parte, McDowell sostiene que el contenido intuitivo es "todo conceptual, en este sentido: está en la intuición en una forma, tal que uno podría hacer que ese mismo contenido, figure en la actividad discursiva" (2009a: 265) y agrega que "es correcto decir que el contenido unificado en las intuiciones es de la misma clase que el contenido unificado en los juicios: esto es, contenido conceptual" (2009a: 264).¿Cómo entender estas declaraciones acerca de la experiencia, específicamente acerca del contenido de la experiencia? A juicio de McDowell (2009a), la clave estaría en el siguiente pasaje de la Crítica de la Razón Pura de Kant:

La misma función que da unidad a las diversas representaciones en un juicio, le da también unidad a la mera síntesis de diversas representaciones en una intuición; [función] que, expresada de manera universal, se llama el concepto puro del entendimiento (Kant, 2007: A79/B104-105).

\footnotetext{
${ }^{12}$ Esta es indudablemente una categorización aristotélica. Los sensibles propios son aquellos aspectos de lo físico que solo puede ser captado por un sentido. El sabor, por ejemplo, es el sensible propio del gusto, el color de la vista, etc. Mediante los denominados sensibles propios, Aristóteles afirmó que podemos captar también otros atributos que también pueden ser captados por otros sentidos. Estos atributos que no son exclusivos de solo un sentido son cinco: movimiento, reposo, número, figura y magnitud.
} 
La unidad del contenido intuitivo de la experiencia no es una unidad Dada (en el sentido del Mito de lo Dado), sino una unidad que es producto de la misma función que unifica el contenido proposicional de los juicios. En este sentido, la unidad "no nos es proporcionada por la sola sensibilidad" (McDowell, 2009a: 264) o no es concebible independientemente de las capacidades que esencialmente son operativas en actividades eminentemente discursivas como nuestros juicios o creencias $^{13}$. Tanto el contenido intuitivo, como el contenido discursivo son, según la nueva teoría de McDowell, contenidos que podríamos considerar como conceptuales, aunque a mi juicio los matices son diferentes. El contenido discursivo es conceptual no solo porque su unidad o enlace es producto de la operación de funciones que esencialmente pertenecen a la facultad discursiva del entendimiento, sino también porque es un contenido proposicional y para McDowell las proposiciones son evidentemente estructuras compuestas de conceptos o modos de presentación. De un modo más débil, el contenido intuitivo es conceptual solo en cuanto en él están en juego algunas capacidades conceptuales asociadas a los sensibles propios y comunes y porque su unidad depende también de la implementación de las mismas funciones que el entendimiento opera al unificar el contenido proposicional de los juicios.

\section{REFLEXIÓN FINAL: CONTENIDO INTUITIVO Y CONCEPTUALISMO DÉBIL}

El conceptualismo acerca de los contenidos de la experiencia perceptual, al menos interpretado de manera estándar, siempre se ha entendido como la confluencia de dos tesis filosóficas de inspiración fregeana. La tesis según la cual los contenidos de la experiencia perceptiva son siempre exhaustivamente proposicionales y la tesis según la cual los elementos constitutivos de estas proposiciones son conceptos o modos de presentación cuyas condiciones de posesión requieren de la satisfacción de ciertos requerimientos vinculados a nuestra capacidad racional para pensar, entre ellos el llamado requerimiento de generalidad de Evans.

El McDowell (2003) clásico de Mente y Mundo es un claro exponente de este conceptualismo estándar. En la búsqueda de un empirismo mínimo en el que

\footnotetext{
${ }^{13}$ Kant agrega además que "todo enlace, ya seamos consciente de él, o no lo seamos...es una acción del entendimiento que designaremos con la denominación general de sintesis" (2007: B130). Este polémico pasaje sugiere que la unidad de la intuición podría perfectamente efectuarse en un dominio subpersonal inconsciente. En tanto tenemos una experiencia consciente de objetos, la unidad ya estaría garantizada previamente por la operación de estas funciones del entendimiento literalmente antes de que nos percatemos desde la perspectiva consciente de la primera persona.
} 
las experiencias pueden jugar un rol de justificación racional para nuestras creencias empíricas McDowell aboga por un conceptualismo sin límites. En todos los casos el contenido de una experiencia perceptual es proposicional y, por lo mismo, su naturaleza es de índole conceptual. El conceptualismo de Mente y Mundo es un conceptualismo fuerte. En él nuestras capacidades conceptuales no solo juegan un rol importante en la configuración de la experiencia en un sentido kantiano, sino que al parecer se desecha por completo el rol (ontológico, fenomenológico o epistemológico) que podría jugar cualquier tipo de representación perceptual de naturaleza no-conceptual. En Mente y Mundo la experiencia perceptual se comprende como una apertura a un mundo pensable, a un mundo de hechos (facts) articulados proposicionalmente donde no hay lugar para componentes que no se manifiesten conscientemente bajo una articulación conceptual.

El nuevo McDowell (2009a) de Avoiding the Myth of the Given claramente propone una teoría diferente acerca de los contenidos de la experiencia perceptual. Para él el contenido de la experiencia ya no es proposicional, sino intuitivo. Sin embargo, el contenido intuitivo de la experiencia, pese a no ser articulado y menos aún proposicional, es conceptual. El fundamento de este conceptualismo radica en que "la misma función que da unidad a las diversas representaciones en un juicio, le da también unidad a la mera síntesis de diversas representaciones en una intuición" (Kant, 2007: A79/B104-105). Evidentemente el conceptualismo que ahora defiende McDowell no es un conceptualismo estándar, ya que las intuiciones kantianas a las que él apela no son entidades proposicionales. La caracterización de su teoría como una propuesta "conceptualista" en el contexto del debate contemporáneo parece depender de cómo está interpretando McDowell la síntesis que, a juicio de Kant, se da en la intuición y de cómo entiende específicamente la relación de dependencia que él mismo afirma existe entre el supuesto carácter conceptual del contenido intuitivo de la experiencia y la naturaleza conceptual de las proposiciones que conforman el contenido de nuestros juicios empíricos ${ }^{14}$.

Me parece importante mencionar como primera reflexión crítica sobre el nuevo conceptualismo de McDowell el hecho de que su formulación no parece ser exhaustiva en términos de la naturaleza de los componentes del contenido de la experiencia.

\footnotetext{
${ }^{14}$ Sugiero dejar a un lado el problema exegético acerca de si McDowell está efectivamente realizando una interpretación adecuada de Kant en beneficio de la propuesta sistemática acerca del contenido intuitivo que a juicio de $\mathrm{McD}$ owell es conceptual. A mi juicio, lo importante en términos históricos es tener claro que el nuevo McDowell se nutre de Kant, fundamentalmente porque el filósofo alemán afirma que es precisamente la misma función la que unifica las diversas representaciones en un juicio y en una intuición sensible. En el contexto de la unidad sintética de lo múltiple en el caso del contenido intuitivo no debemos nunca olvidar que los conceptos son las reglas que gobiernan esta síntesis y no aquello que es unificado. Para una interesante discusión de la lectura que hace McDowell de la Crítica de la Razón Pura de Kant recomiendo ver Gauker (2011:70-85) y Hannah (2005).
} 
La manera en que McDowell (2009a) introduce el contenido intuitivo parece ser consistente plenamente con la tesis de que no todos los elementos constitutivos de ese contenido intuitivo son conceptos, permitiendo que existan componentes que sean en principio solo conceptualizables. Detengámonos en un par de citas en las que McDowell, a ratos de manera característicamente oscura, intenta especificar la clase de relación existente entre las capacidades conceptuales y los contenidos de la experiencia:

¿Si el contenido intuitivo no es discursivo, por qué continuar insistiendo que es conceptual? Porque cada aspecto del contenido de una intuición está presente en una forma en la que ya es adecuado para ser el contenido asociado a una capacidad discursiva, aunque no esté -al menos no aún- realmente asociado así. Aquello es parte de la fuerza de decir, con Kant, que aquello que le da unidad a las intuiciones es la misma función que le da unidad a los juicios (McDowell, 2009a: 264).

Sin embargo, el contenido de una intuición es todo conceptual, en este sentido: está en la intuición en una forma, tal que uno podría hacer que ese mismo contenido, figure en la actividad discursiva" (McDowell, 2009a: 265).

Los contenidos de los juicios son contenidos esencialmente discursivos, es decir, proposiciones compuestas de conceptos. McDowell afirma que los contenidos intuitivos de la experiencia son utilizables por los juicios empíricos, aunque no estén aun realmente asociados con una actividad discursiva, es decir, aunque no todos los aspectos o elementos estén de hecho asociados a un concepto. A mi juicio, las afirmaciones de McDowell dejan abierta la posibilidad de una lectura menos demandante respecto de la naturaleza de los componentes de los contenidos intuitivos. Uno podría interpretar que no todos los elementos de un contenido deben ser conceptos o estar de hecho asociados o explotados por el ámbito discursivo ${ }^{15}$. El conceptualismo en juego se fundaría más bien en la forma específica en que los aspectos componen la intuición, una forma que en principio permite que estos aspectos sean conceptualizables. Tal como ha afirmado recientemente Crane, "es útil pensar a acerca de la conceptualización como "explotación discursiva”, de aquello que está allí para ser conceptualizado pero no necesariamente explotado de tal forma” (2013: 241). Si esto es así, no veo inconveniente alguno en afirmar que el nuevo conceptualismo de McDowell es un conceptualismo débil que en principio es compatible con la existencia de componentes no-conceptuales del contenido intuitivo, algo que ciertamente se distancia drásticamente de los estándares clásicos de Mente y Mundo.

15 Para una lectura diferente de los textos de Kant, según la cual en ellos estaría de hecho ya sembradas las semillas del no-conceptualismo contemporáneo, sugiero revisar Hannah (2008), Allais (2009), Lazos (2012) y Peláez (2013). 
¿Por qué McDowell insiste que el contenido intuitivo es todo conceptual si de hecho no todos sus componentes necesitan estar de hecho asociados a una actividad discursiva? McDowell ciertamente afirma esto, pero agrega que hay que entenderlo en un sentido muy específico, en "este sentido" (2009a: 265). La clave estaría en el hecho de que ciertas capacidades conceptuales, las mismas que operan en la unificación de los contenidos discursivos, se actualizarían también en el proceso de unificación de la intuición. Esto es precisamente lo que McDowell expresa en lo que él denomina un sentido específicamente kantiano. Es precisamente en este contexto donde surge una segunda reflexión crítica en torno al nuevo conceptualismo de McDowell. Si tomamos en serio la fuente kantiana de inspiración es evidente que los conceptos que gobiernan la síntesis de la intuición, es decir, las reglas que gobiernan esta síntesis son conceptos formales o puros del entendimiento: categorías kantianas. No es para nada evidente que estas categorías kantianas puedan codificarse con claridad en términos de lo que hoy entendemos como un concepto en el contexto neofregeano en el que el mismo McDowell se desenvuelve, es decir, como un componente de una proposición cuya posesión se especifica en términos de la implementación de un conjunto de capacidades como, por ejemplo, nuestra capacidad para reconocer y realizar inferencias racionales ${ }^{16}$.

McDowell (2009a) no explica con claridad cómo es que cualquier concepto podría figurar realmente -como un componente- en el contenido intuitivo de la experiencia. De hecho, ni siquiera especifica en qué sentido los conceptos puros del entendimiento kantiano o, en su defecto, "las capacidades conceptuales asociadas con los conceptos de sensibles propios y comunes" (McDowell, 2009a: 260) pueden de hecho conformar el contenido de una experiencia y no solo figurar como reglas de síntesis. El conceptualismo en juego no parece ser algo que opere en un dominio metafísico o constitutivo del contenido de la experiencia, sino más bien una tesis que se menciona como producto de la falta de autonomía o dependencia que de hecho tiene la unidad de la intuición respecto de los conceptos que cumplen esta misma función en la actividad discursiva y proposicional del juicio. En este punto tiendo a concordar con la tesis recientemente planteada por Toribio (2008), según la cual el contenido intuitivo no es esencialmente conceptual, es decir, no es una

\footnotetext{
${ }^{16}$ Si lo que McDowell tiene hoy en mente es la actualización de categorías kantianas como condición de posibilidad de la unidad sintética de la multiplicidad en la intuición, entonces su propuesta no se enmarca con suficiente claridad dentro de lo que hoy denominaríamos "conceptualismo" en el contexto neofregeano predominante. En la segunda edición de la deducción trascendental Kant sostiene que las categorías son "conceptos de un objeto en general, mediante los cuales la intuición de éste se considera como determinada respecto de una de las funciones lógicas [requeridas] para los juicios" (2007: B128). No es claro que esto implique que las categorías o conceptos puros sean componentes exhaustivos de los contenidos intuitivos, sino más bien expresión de las funciones unificadoras de los mismos. Esto requiere por lo menos de una aclaración por parte de McDowell.
} 
tesis metafísica acerca de la naturaleza de todos los elementos que componen los contenidos de la intuición, sino más bien solo conceptual en "carácter"17.

La conceptualidad en juego no parece ser una propiedad monádica propia del contenido intuitivo, sino más bien una propiedad diádica relativa a una relación explícita de dependencia existente entre el contenido intuitivo y el contenido discursivo. A mi juicio, esta relación de dependencia que neutraliza cualquier esbozo de autonomía del contenido de la experiencia respecto del contenido discursivo es la clave para interpretar el nuevo conceptualismo de McDowell. Es en este sentido que él mismo afirma que "no podríamos tener intuiciones, con sus formas específicas de unidad, si no pudiésemos realizar juicios, con sus correspondientes formas de unidad" (2009a: 264).

Para el nuevo McDowell no podríamos tener experiencias con contenido intuitivo en las que representamos conscientemente objetos, si no fuésemos capaces de tener pensamientos de naturaleza proposicional acerca de los mismos. La pérdida de autonomía del contenido de la experiencia respecto de los contenidos de los juicios y creencias se explica mediante un postulado trascendental kantiano. La unidad de lo múltiple en la intuición y la unidad de la representación en un juicio requieren de la operación de las mismas reglas de síntesis, esto es, al menos de la actualización de ciertos "conceptos". Sin embargo, afirmar que la unidad del contenido intuitivo de la experiencia no es producto de la mera sensibilidad parece ser compatible con la posibilidad de que elementos conceptualizables del contenido intuitivo, no sean de hecho componentes conceptuales del contenido de la experiencia en un momento particular, es decir, de facto asociados con aspectos del contenido proposicional discursivo. El nuevo McDowell debilita cualquier compromiso ontológico sustantivo acerca de todos los elementos que de hecho componen el contenido mismo de la intuición. Si estoy en lo correcto, es un conceptualismo débil, ya que los contenidos de la experiencia no tienen que estar necesariamente compuestos de modo exhaustivo por conceptos, de un modo análogo a como de hecho sí lo están los contenidos de los juicios empíricos y las creencias que formamos a partir de esas experiencias ${ }^{18}$.

\footnotetext{
${ }_{17}$ Toribio afirma que en la nueva propuesta de McDowell "el contenido de las experiencias perceptuales no es conceptual. El contenido de las experiencias perceptuales es intuitivo. El contenido intuitivo es, sin embargo, conceptual en carácter no debido a la estructura y naturaleza de los constituyentes del contenido mismo, sino porque las capacidades cognitivas cuyo ejercicio explica la unidad de las experiencias perceptuales son las mismas capacidades conceptuales cuyo ejercicio de cuenta de la unidad de los juicios" (2008:6).

${ }^{18}$ Deseo agradecer a Søren Overgaard del Center for Subjectivity Research de la Universidad de Copenhagen por su cordial invitación a asistir al workshop "Experiential Reasons".
} 


\section{REFERENCIAS}

Allais, Lucy. "Kant, Non-Conceptual Content and the Representation of Space", Journal of the History of Philosophy 47 / 3 (2009): 383- 413.

Bermúdez, José Luis. "Peacocke’s argument against the autonomy of nonconceptual representational content.” In Y. H. Gunther (Ed.), Essays on nonconceptual content. Cambridge: The MIT Press, (1994): 293-307.

Brewer, Bill. Perception and Reason. Oxford, New York: Clarendon Press - Oxford University Press, 1999.

Brewer, Bill. "Perceptual Experience has Conceptual Content." In Ernest Sosa \& Matthias Steup, (Eds.), Contemporary Debates in Epistemology. Oxford: Blackwell, (2005): 217-230.

Crane, Tim. "The Given". In Josep Schear (ed.), Mind, Reason and Being-In-TheWorld: The McDowell-Dreyfus Debate. London: Routledge, (2013): 229-249.

Davidson, Donald. "A Coherence Theory of Truth and Knowledge." In Donald Davidson (Ed.), Subjective, Intersubjective, Objective. Oxford: Clarendon Press, (2001): 137-153.

Dreyfus, Hubert. Overcoming the Myth of the Mental: How Philosophers Can Profit from the Phenomenology of Everyday Expertise (APA Pacific Division Presidential Address 2005). In Proceeding and Addresses of the APA 79: 2, November 2005.

Dreyfus, Hubert. "The Return of the Myth of the Mental”, Inquiry 50 / 4 (2007a): 352-365.

Dreyfus, Hubert."Response to McDowell”, Inquiry 50 / 4 (2007b): 371-377.

Evans, Gareth. The Varieties of Reference. Oxford, New York: Clarendon Press Oxford University Press, 1982.

Gauker, Christopher. Words and Images. An Essay on the Origins of Ideas. Oxford, New York: Clarendon Press - Oxford University Press, 2011. 
Hannah, Robert. "Kant and Non-Conceptual Content", European Journal of Philosophy 13 (2005): 247-290.

Hannah, Robert. "Kantian Non-conceptualism”, Philosophical Studies 137 /1 (2008): 41-64.

Kant, Immanuel. Crítica de la Razón Pura (Trad. Mario Caimi). Buenos Aires: Colihue, 2007.

Lamme, Victor. "Why visual attention and awareness are different", Trends in Cognitive Sciences 7 (1), (2003): 12-18.

Lazos, Efraín. “¿Es Kant un conceptualista?” En Pedro Stepanenko (Ed.), La primera persona y sus percepciones. México: UNAM, 2012.

McDowell, John. Mente y Mundo. Salamanca: Editorial Sígueme, 2003.

McDowell, John. "What Myth?”, Inquiry 50 / 4 (2007a): 338-351.

McDowell, John. "Response to Dreyfus", Inquiry 50 / 4 (2007b): 366-370.

McDowell, John. Avoiding the Myth of the Given, Gareth Evans Memorial Lectures delivered at Oxford, 2007c.

McDowell, John. "Avoiding the Myth of the Given". In John McDowell (Ed.), Having the World in View. Cambridge: Harvard University Press, (2009a): 256-272.

McDowell, John. "Experiencing the World". In John McDowell (Ed.), The Engaged Intellect. Cambridge: Harvard University Press, (2009b): 243-256.

Papineau, David. "Sensory Experience and Representational Properties" Proceedings of the Aristotelian Society. The 106th Presidential Address 114/1 (2014): 1-33.

Peacocke, Christopher. "Does Perception have a nonconceptual content?", Journal of Philosophy 98 (2001): 239-264.

Peláez, Álvaro. "Espacio, movimiento y contenido no-conceptual en la filosofía de la experiencia de Kant", Signos Filosóficos XV / 30 (2013): 45-69. 
Pryor, Jim. "There is immediate justification". In Ernest Sosa \& Matthias Steup (Eds.), Contemporary Debates in Epistemology. Boston: Willey Blackwell, (2005): 181-201.

Raftopoulos, Anastasios. Cognition and Perception. Cambridge, MA: MIT Press, 2009.

Toribio, Josefa. "Intuitional Content, Nonconceptual Content and Perceptual Justification". In Chase B. Wrenn (Ed.), Naturalism, Reference, and Ontology: Essays in Honor of Roger F. Gibson. New York: Peter Lang Publishing Inc., (2008): 93-104.

Travis, Charles. "The Silence of the Senses", Mind 113 / 449, (2004): 59-94.

Travis, Charles. "Reason's Reach", The European Journal of Philosophy 15 /2, (2007): 204-224.

Tye, Michael. "Nonconceptual content, richness, and fineness of grain". In Tamar Gendler \& John Hawthorne (Eds.), Perceptual experience: Oxford University Press, (2006): 504-530.

Wright, Crispin. "Mind and World by John McDowell", Philosophy and Phenomenological Research 58 / 2 (1998): 395-402. 